\title{
Observations of omega bands using an imaging riometer
}

\author{
A. J. Kavanagh, J. A. Wild, and F. Honary \\ Department of Communication Systems, InfoLab21, Lancaster University, Lancaster LA1 4WA, UK
}

Received: 22 August 2008 - Revised: 13 October 2009 - Accepted: 30 October 2009 - Published: 6 November 2009

\begin{abstract}
We present a case study of an omega band current system from 11 May 1998 using data from the Imaging Riometer for Ionospheric Studies (IRIS) in Finland. For the first time, images of a substorm-related omega band in cosmic noise absorption are shown. The substorm in question was one of a string that occurred on that day; inspection of geostationary satellite data indicates that this was a sawtooth event. Using a previously established statistical relationship, the IRIS data is used to provide maps of Hall conductance and compared with previous estimates utilising both HF and VHF coherent-scatter radars. Discrepancies are discussed with reference to precipitation spectrum and the geometry of the experimental set-up. The imaging riometer data provides a higher spatial resolution than the combined magnetometerradar pairing for determining the Hall conductance and can also be used to identify the extent of the precipitation in the absence of optical data.
\end{abstract}

Keywords. Ionosphere (Particle precipitation) - Magnetospheric physics (Current systems; Magnetospheric configuration and dynamics)

\section{Introduction}

The precipitation of energetic ( $>30 \mathrm{keV}$ ) electrons in the auroral zones is strongly connected to substorm activity and the resulting enhanced electron density in the lower ionosphere leads to cosmic noise absorption (CNA) which can be used as a proxy for this precipitation. Measurements of CNA have often been used to study the precipitation during and following substorm activity, both on the night side and the subsequent eastward drift of electrons in the inner magnetosphere (e.g. Hargreaves et al., 1975, 1997; Ranta et al., 1981; Baker et al., 1981; Kavanagh et al., 2002; Span-

Correspondence to: A. J. Kavanagh (a.j.kavanagh@lancaster.ac.uk) swick et al., 2007). Around dawn CNA often displays periodic variations in the $\mathrm{mHz}$ frequency range. Much of this structure can be attributed to the modulation of precipitation by ULF (ultra low frequency) geomagnetic pulsations (e.g. Senior and Honary, 2003; Spanswick et al., 2005); however, longer quasi-periodic structures that display eastward motion at the convection speed also appear (e.g. Hargreaves and Berry, 1976; Kavanagh et al., 2002; Makarevitch et al., 2004). The exact cause of these structures is unknown and it is not clear whether the same process drives the long-period variations in precipitation in all cases.

One potential cause of these precipitation structures is the propagation of auroral omega bands, which occur during the recovery phase of substorms (Akasofu, 1974; Saito, 1978; Rostoker and Barichello, 1980). These are eastward drifting (Luhr and Schlegal, 1994) current systems that are mostly (but not strictly) stationary in time (Amm et al., 2005) and which produce luminous auroral tongues, the dark gaps between forming the shape of an inverted $\Omega$ (hence the name). Passage of the current system generate magnetic pulsations on the ground (Saito, 1978; Kawasaki and Rostoker, 1979; Opgenoorth et al., 1983) that are observed by magnetometers as Ps6 pulsations (period $=5-40 \mathrm{~min}$ ), dominant in the azimuthal component, caused by the formation of Hall currents. Although predominantly associated with the substorm recovery phase (Opgenoorth et al., 1994) omega bands have also been suggested as a storm-time phenomena following sudden impulses (Nielsen and Honary, 2000) and during steady magnetospheric convection (e.g. Solovyev et al., 1999).

A case study of periodic CNA enhancements close to dawn is presented from 11 May 1998; this is the first identified observation of substorm-related omega bands in imaging riometer data. This event has already been investigated using magnetometer and radar data by Wild et al. (2000), the combination of which provided a means of determining the associated conductance. Estimates of the mesoscale spatial distribution of the hall conductance based on the

Published by Copernicus Publications on behalf of the European Geosciences Union. 


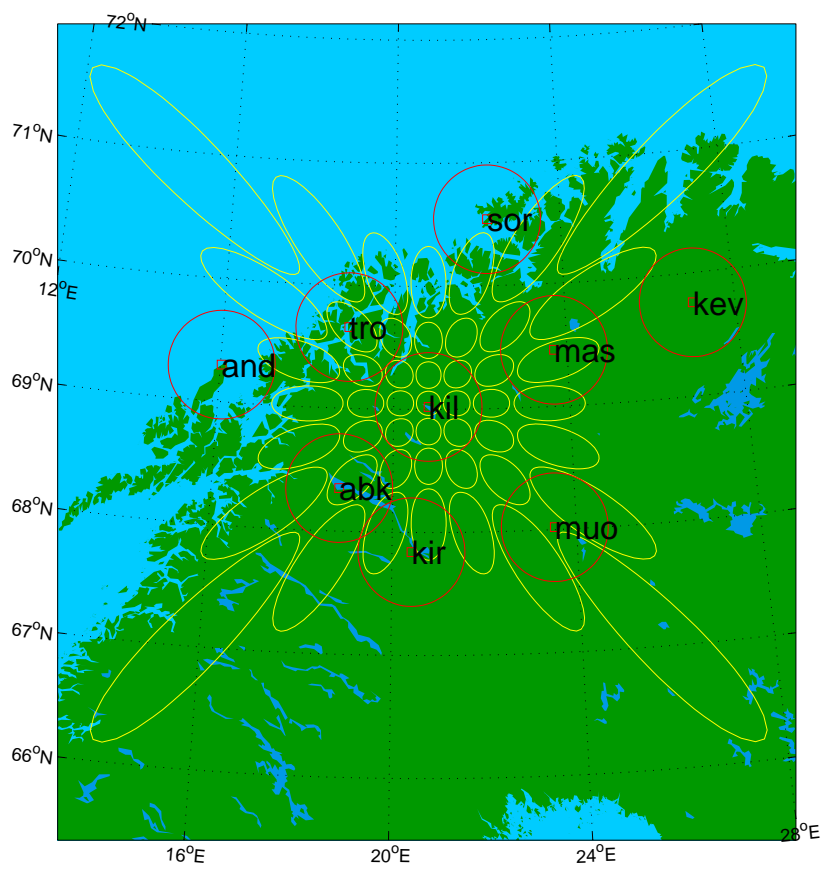

Fig. 1. Beam projection of IRIS at $90 \mathrm{~km}$ altitude (yellow) and estimated fields of view of selected magnetometers from the IMAGE chain (red circles). Coordinates are in geographic latitude and longitude.

CNA measurements are made and the magnitude is shown to be reasonably consistent with past observations during this event. The transition from pulsed CNA to pole-ward propagating patch following the onset of a second substorm is shown; this is attributed to the local break-down of the current system as a population of freshly injected electrons gradient-curvature drifts into the field of view of the instrument.

\section{Instrumentation}

Cosmic Noise Absorption data have been taken from the Imaging Riometer for Ionospheric Studies (IRIS) at Kilpisjärvi, Finland $\left(69.05^{\circ} \mathrm{N}, 20.79^{\circ} \mathrm{E}\right.$ ) (Browne et al., 1995). This uses a phased array to generate 49 imaging beams with widths of $\sim 20 \mathrm{~km}$ in the D layer of the ionosphere; each beam is sampled every second though in the following study we use a minimum of $10 \mathrm{~s}$ averages of the data. A single widebeam $(\sim 100 \mathrm{~km}$ width $)$ is co-located with IRIS. Figure 1 shows the beam pattern of IRIS (projected at $100 \mathrm{~km}$ altitude) with estimates of the fields of view $(100 \mathrm{~km}$ radius at $100 \mathrm{~km}$ altitude for raw data) of several of the magnetometers used in this study. Seven of the magnetometers observe substantially within the IRIS beam pattern allowing for direct comparisons between the data sets. These data have been supplied by the International Monitor for $\mathrm{Au}$ roral Geomagnetic Effects (IMAGE) magnetometer stations
(Lühr et al., 1998) and the Sub-Auroral Magnetometer Network (SAMNET) (Davison and Orr, 1989). The resolution of the magnetometers can be improved (to about $50 \mathrm{~km}-$ Amm et al., 2005) through application of the "magnetic field upward continuation" procedure (Untiedt and Baumjohann, 1993); this has not been applied to the data presented so as to remain consistent with past estimates of the conductance in this event. In this study the perturbations have been derived by removing a "quiet-time" baseline from the magnetometer data; this is calculated by identifying the quietest days (where local $K<3$ ) around the interval of interest (one month either side of the event in question). From these data the most likely value in each time step is calculated via a probability density estimate; the resulting curve is smoothed using a SavitzkyGolay filter and subtracted from the data for the period in question. This is a different technique to the one employed by Wild et al. (2000) but the resulting magnetic perturbations are exceptionally similar.

Direct observations of substorm related electron injections and field dipolarization at geosynchronous orbit have been provided by the LANL (Los Alamos National Laboratory) and GOES (Geosynchronous Operational Environmental Satellites) satellites. Particle data were taken from the SOPA (Synchronous Orbit Particle Analyzer) instrument, (LANL) in the seven energy channels spanning 50 to $315 \mathrm{keV}$ (Belian et al., 1992; Reeves et al., 1997). Magnetic field measurements have been provided by twin-fluxgate magnetometers on GOES-8 and GOES-9 (Singer et al., 1996). The inclination of the magnetic field relative to the GSM (Geocentric Solar Magnetospheric) equatorial plane has been calculated.

\section{Observations}

On 11 May 1998 a Ps6 pulsation was observed by the IMAGE magnetometer chain between 01:30 UT and 03:30 UT (the magnetic local time of the magnetometers is approximated by $\mathrm{MLT} \approx \mathrm{UT}+2$ ). These data have been previously presented by Wild et al. (2000) but we will summarize the basic observations. Five clear wavelengths with a period (frequency) of $\sim 17 \mathrm{~min}(0.98 \mathrm{mHz})$ were observed, shown in Fig. 2 of Wild et al. (2000); the peak to peak amplitude of the pulsation in the Y-component maximises around 02:20 UT $(\sim 130 \mathrm{nT}$ at SOR). Calculations of the delay between AND and SOR suggest an eastward propagation with a mean velocity of $750 \mathrm{~ms}^{-1}$. The pulsation was also visible in the line of sight velocity data of both the CUTLASS (Greenwald et al., 1995) and STARE (Greenwald et al., 1978) radars; these indicate that the omega band is confined between $\sim 66^{\circ}$ and $74^{\circ}$ magnetic latitude. The field of view of IRIS lies between magnetic latitudes of $65.2^{\circ}$ and $66.8^{\circ}$ covering an equatorward portion of the ionosphere affected by the pulsation. Unfortunately no optical data were available for this period. 


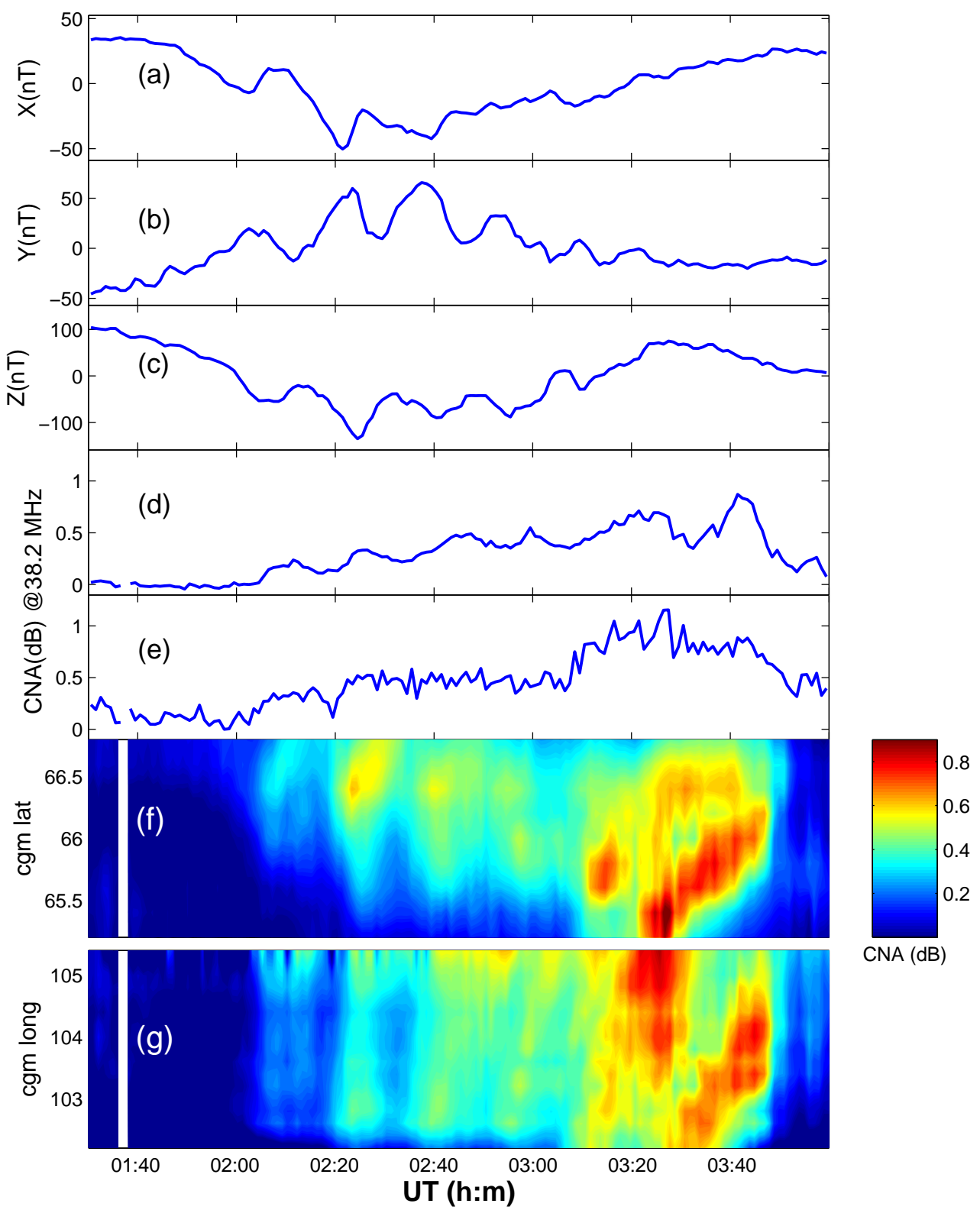

Fig. 2. Data from the magnetometer at Kilpisjärvi (KIL) (a-c) and corresponding riometer data from IRIS (d-g) showing signatures of the Ps6 pulsation on 11 May 1998. The central beam of IRIS (d) shows the pulsation whereas the co-located widebeam (e) does not. Panel (f) is a keogram of the latitudinal variation of CNA taken at the longitude of the Troms $\emptyset$ magnetometer where the pulsation is strongest. Panel (g) presents an east-west slice across the IRIS field of view where the eastward motion of the precipitation structures becomes clear.

Figure 2 shows data from both the magnetometer and IRIS at Kilpisjärvi. The top three panels $(\mathrm{a}-\mathrm{c})$ show the three magnetic components; as expected the pulsation is clearest in the $\mathrm{Y}$ and $\mathrm{Z}$ components. The next two panels $(\mathrm{d}-\mathrm{e})$ show the CNA from the central narrow beam $\left(\sim 12^{\circ}\right.$ width) of IRIS (d) and the co-located widebeam $\left(\sim 90^{\circ}\right.$ width) (e). The pulsation is clear in the narrow beam data and appears on a background of increasing absorption showing a distinct phase delay when compared with the magnetometer data. The widebeam integrates the absorption across a similar portion of the sky to the magnetometer and although the CNA increases from $0.2 \mathrm{~dB}$ to $1.2 \mathrm{~dB}$ there is less evidence of the Ps6 pulsation. Panel (f) in Fig. 2 is a keogram constructed from a latitudinal slice across the IRIS field of view at the longitude of the Troms $\varnothing$ magnetometer where the Ps6 pulsation is strongest. It is clear that the precipitation pulsation has highest amplitude at the poleward end of the array but after 03:10 UT there is an additional increase in CNA particularly to the south of the array. This coincides with an increase in low power ULF Pc4 fluctuations in both the magnetic 


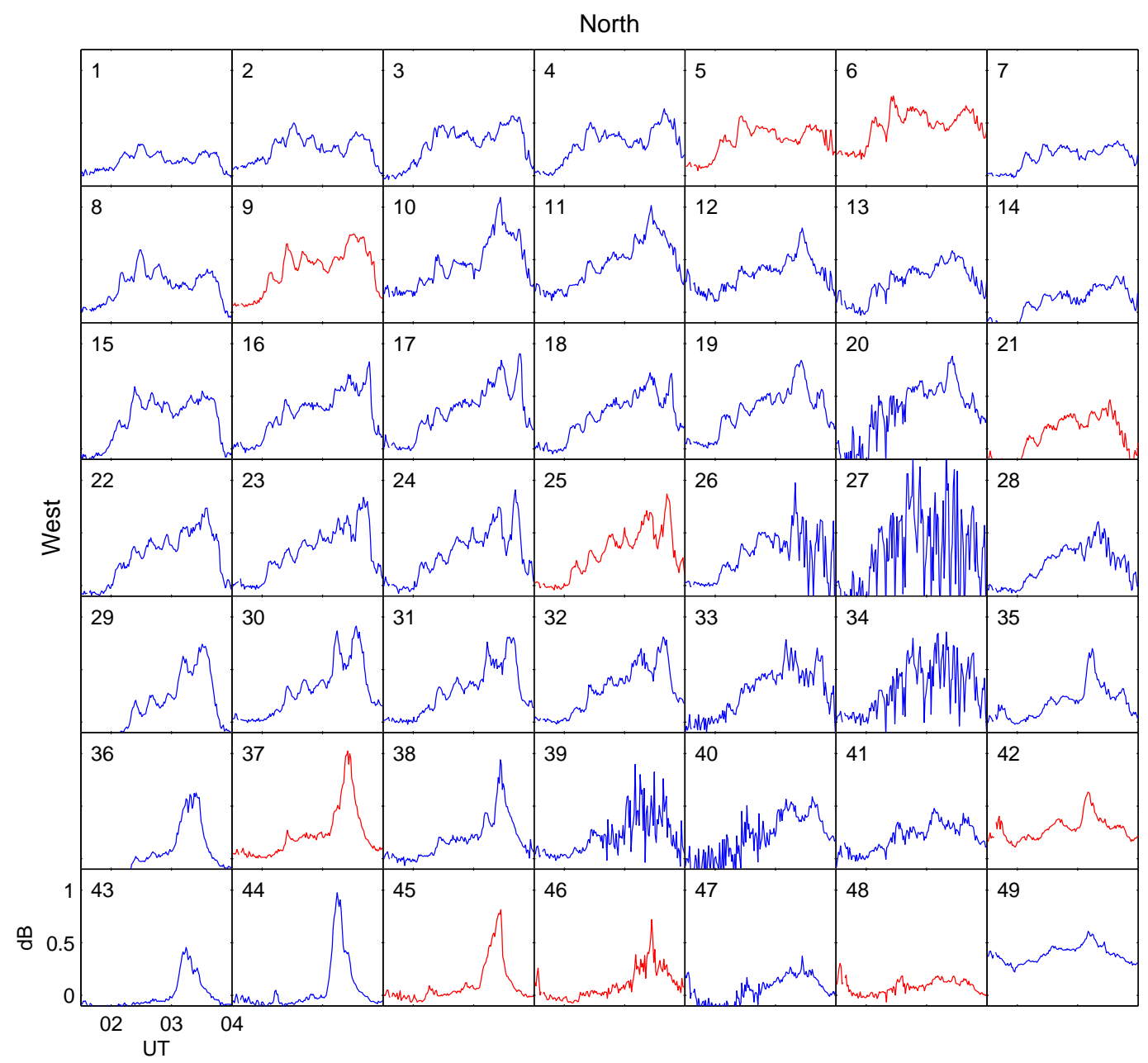

Fig. 3. Data from all 49 beams of IRIS for the duration of the pulsation. Note that the data in the four corner beams $(1,7,43,49)$ should be ignored due to the small elevation angles and large side-lobes. Scintillation is clear in beams 20, 26, 27, 33, 34, 39 and 40, caused by the passage of a radio star through the beam. Red lines indicate those beams closest to the magnetometer sites.

(particularly X component) and absorption data. Panel (g) is a keogram in longitude taken across the centre of the field of view. The eastward propagation of the omega bands is evident, as is the eastward motion of the feature after 03:10 UT; the steeper gradient at later times indicates a higher apparent velocity.

Figure 3 shows time series of all 49 beams of IRIS during the pulsation from 01:30 to 04:00 UT. Geomagnetic north is at the top of the plot with east to the left. The number of each beam is labelled in the top left hand corner and those traces presented in red are from beams that lie underneath the estimated fields of view of the IMAGE magnetometers. Note that the four corner beams $(1,7,43$ and 49$)$ have elevation angles of $\sim 21^{\circ}$ with very large side-lobes; consequently they are less reliable than the remaining beams and should be ignored. Several of the beams $(20,26,27,33,34,39$ and 40) suffer from large variations in signal strength at various points during the period; this is caused by radio stars (in this case Cassiopeia) appearing within the beam or a side lobe. Radio waves from the strong point source pass through irregularities in the F-layer ionosphere introducing scintillation into the signal and masking changes due to variations in electron density in the D-layer.

The amplitude of the pulsation is largest in the north and west of the IRIS field of view, which is in the direction of the magnetic pole; the amplitude is vanishingly small in the equatorward beams (e.g. beam 33 onwards). CNA increases steadily with time except in the more equatorward beams where an abrupt increase occurs only after 03:00 UT. This increase is strongest towards the equator but is visible across the whole array. The keograms in Fig. 2 indicate that the post 03:00 UT enhancement displayed both a poleward and eastward motion (in corrected geomagnetic coordinates). Figure 3 shows that at the poleward edge of this enhancement it overlaps the pulsation and the subsequent decrease in CNA shows that the Ps6 pulsation has ceased within the IRIS field of view. 

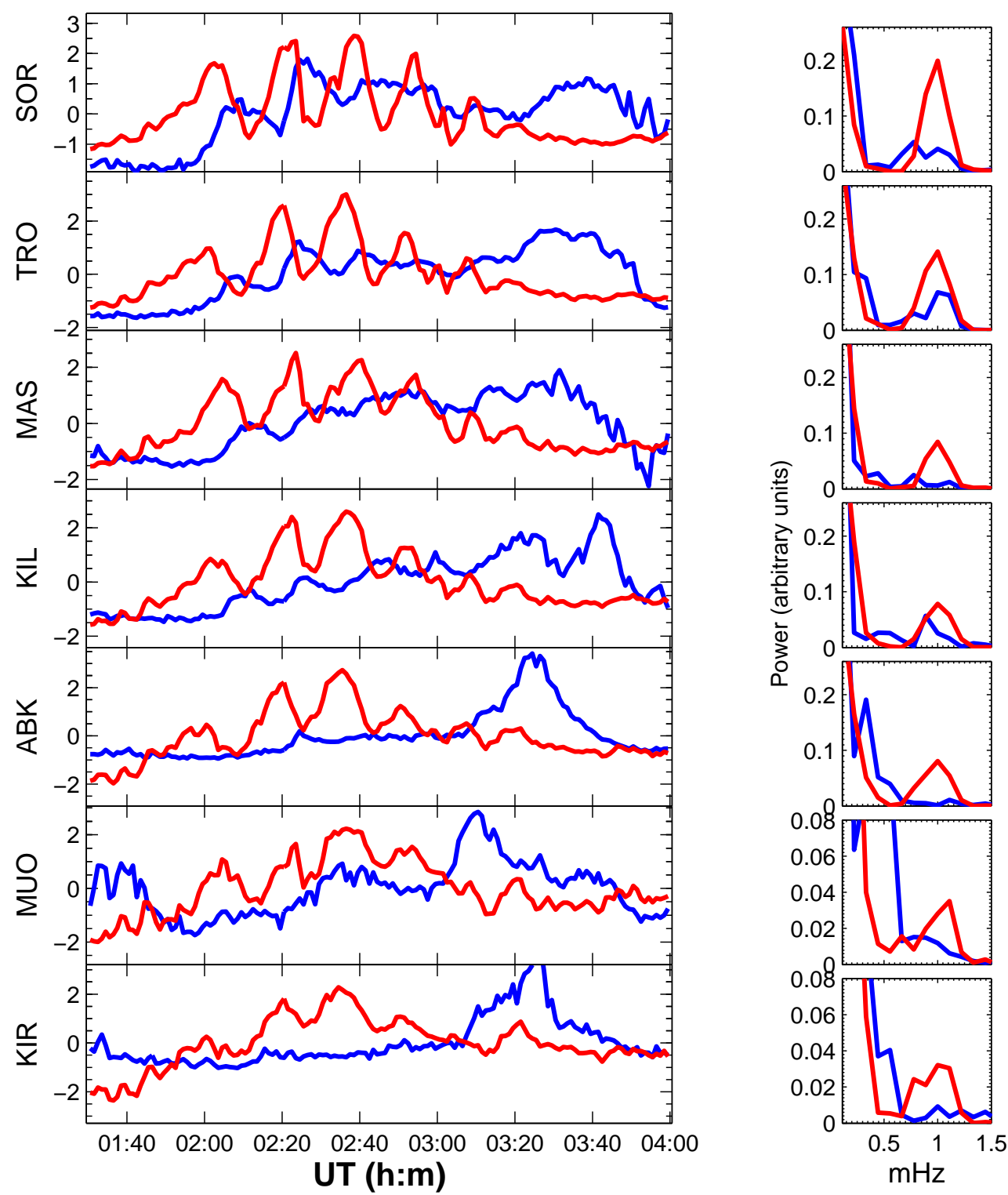

Fig. 4. Comparison of the co-located riometer (blue) and magnetometer (red) Y-component data. The data in the left hand column have been normalized to units of standard deviation to ease comparison (subtract the mean for each time series and divide by the standard deviation). The results of a Fourier analysis of the interval are in the right-hand column showing the power from 0 to $1.5 \mathrm{mHz}$. Note the change in power scale at MUO and KIR.

\section{Discussion}

Nielsen and Honary (2000) reported IRIS observations of a series of eastward propagating CNA patches that they identified as an omega-band-like structure. In that instance the current-system was associated with a sudden commencement rather than a substorm (the authors failed to identify substorm activity during the SC) and the authors speculated that the observed phenomenon was associated with rapid transport of large amounts of energy to the dayside of the Earth. Kawasaki and Rostoker (1979) first identified Ps6 pulsations in wide-beam riometer data and established a good correlation with the D-component of the magnetometer; they suggested that the CNA was caused by electrons with energy between 5 and $20 \mathrm{keV}$ with a flux of $\sim 10^{6}-10^{8} \mathrm{~cm}^{-2} \mathrm{~s}^{-1} \mathrm{sr}^{-1}$. Later authors considered riometer observations when developing models of the current structure in omega bands (e.g. Gustafsson et al., 1981; Opgenoorth et al., 1983). This paper presents the first observation of a substorm-related omegaband structure in imaging riometer data. The imaging capability of IRIS allows for meso-scale observations of the precipitation improving upon the point measurements available 

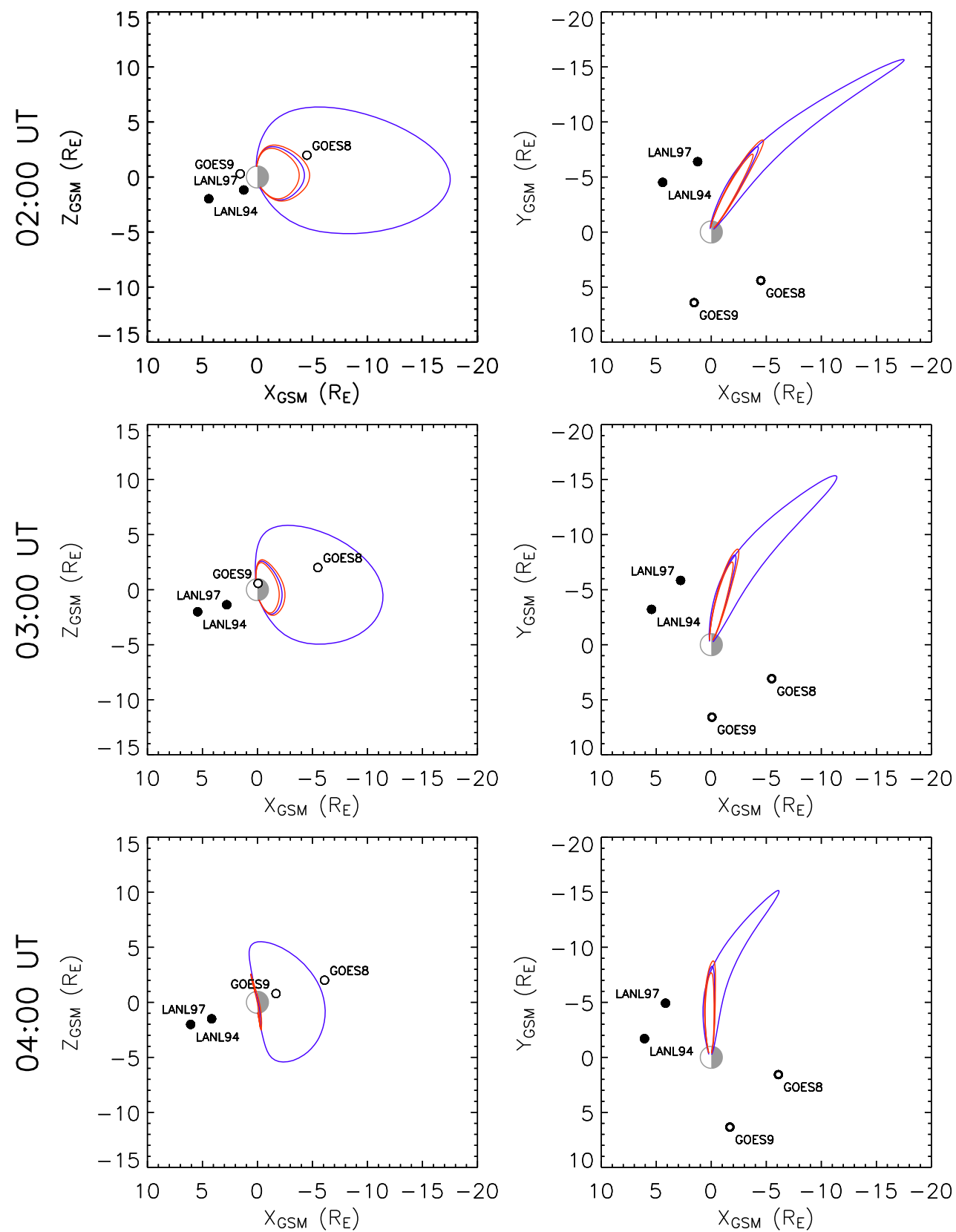

Fig. 5. Locations of the two LANL and GOES satellites in GSM coordinates at three times. The blue lines show the mapped field lines (from T96 model) that mark the latitudinal boundaries of the pulsation determined from HF radar and magnetometer observations. The red lines show the maximum extent of the region of enhanced precipitation (within the IRIS field of view).

from wide-beam riometers. This is particularly useful in the absence of optical data even with the comparatively reduced field of view of imaging riometers.

\subsection{Extent of precipitation region}

Figure 4 shows a direct comparison between the magnetometer Y-component and the riometer response to the Ps6 pulsation. The left hand column shows the magnetometer data (red line) from those instruments within the IRIS field of view with the absorption data from the corresponding riometer beams (blue line); multiple riometer beams that are mostly covered by a single magnetometer field of view have been averaged together. The data are normalized into units of standard deviation to simplify comparison. The right-hand column shows the power spectral density (in arbitrary units) of the magnetometer and riometer time series. Note that for SOR, TRO and KIL there is power around the frequency of 
the Ps6. For the more equatorward pairings (ABK, MUO and KIR) there is much less power, though more from the magnetometer than the riometer. Of particular note is KIR, where the left hand panel shows a clear wave signature that corresponds with an increase in power around $0.98 \mathrm{mHz}$. The riometer signal hints at a small increase but it is not visible in the time series. The discrepancy is readily explained by the nature of the measurements. The riometer is only sensitive to electron density enhancements directly within its beam (or to a much lesser extent, its side lobes); the magnetometer has a field of view that is estimated to be about $100 \mathrm{~km}$ in the E layer but in fact will detect strong signals from a significant distance away. Thus the magnetometer at KIR is responding to a current structure some distance to the north whereas there is little overhead electron precipitation. This ably demonstrates the added value of using the imaging riometer to study discrete current systems such as omega bands since it helps to place other observations in proper context.

The extent of the current system also helps to explain the failure of the wide-beam riometer to detect the Ps6 pulsation (Fig. 2). The wide-beam riometer at IRIS has a beam width approaching $90^{\circ}$ which encompasses all beams in the array except for the outer edge (see Fig. 1). This integrates across regions of the ionosphere that are not experiencing increased precipitation and so leads to damping of the Ps6 signal in the wide-beam data. Inspection of Fig. 3 shows that several of the eastward-pointing beams (20,27, 34, 39 and 40) are experiencing scintillation during the pulsation. This further explains the lack of Ps6 and the increased noise in the widebeam compared to the narrow central beam.

\subsection{Magnetospheric context}

Using the Tsyganenko-96 (T96) model we can map the ionospheric footprint of the omega-band structure out into the magnetosphere in order to estimate the source region of the electrons that drive the omega-band structure. Figure 5 shows the results of such a mapping for 02:00 (top), 03:00 (middle) and 04:00 UT (bottom) with projections onto the GSM X-Z (left column) and X-Y (right column) planes. The blue traces indicate the field lines that link to the lower and upper latitude boundaries of the Omega-bands as determined from HF radar and magnetometer observations (Wild et al., 2000). It must be noted that the magnetometer is responding to the location of the Hall currents associated with the field-aligned currents (FAC) within the precipitation structure and so do not exactly define the precipitation structure. Consequently the red trace is the estimated maximum extent of the region of enhanced precipitation that appears after 03:00 UT. In this case T96 has been driven using the data from the ACE (Advanced Composition Explorer) satellite with a simple time-lag derived from the solar wind velocity measurement. At 02:00 UT when the omega band was approaching its peak intensity (at SOR) the T96 model sug- gests that the source region for the precipitation lies between a tail-like field-line that stretches $16 R_{E}$ towards the dawn flank and $17 R_{E}$ down-tail and a more dipolar field line with apex at $8 R_{E}$ towards the flank The extent of the precipitation enhancement that appears after 03:00 UT is marked in red; according to T96 this part of the IRIS array consistently maps onto field-lines between $\sim 7.7$ and $8.8 R_{E}$.

It must be noted that around the time of this event a number of substorms occurred and the standard T96 model cannot reproduce the dynamic behaviour of the system at these times. However, the local time of the observations (away from the main area of tail activity) and the coincidence with past observations give confidence in the model results.

Although there was no direct observation of the omegaband source region we can still examine the magnetospheric context in which the associated current system formed. Wild et al. (2000) used Greenland magnetometers to identify a number of substorm onsets around the time of the magnetometer observations. In Fig. 6 we show the SOPA particle flux data from two of the LANL satellites along with the measurements of the inclination of the geomagnetic field from the GOES 8 and 9 satellites. It is immediately clear that a number of electron injections and field dipolarizations occurred throughout the day; these are indicative of a series of substorms onsets that repeat with a periodicity of $\sim 2.2 \mathrm{~h}$. This is suggestive of a Sawtooth event (e.g. Borovsky et al., 2001; Henderson et al., 2002; Kavanagh et al., 2007). Thus the observation of traditional substorm-like characteristics (i.e. an omega band) in a "tooth" is further evidence that sawtooth events comprise a series of substorms. Both GOES satellite are westward (duskward) of IRIS whereas the LANL satellites lie eastward (dawnward) of the riometer; hence the electron fluxes show a dispersed signature since the measurements are made at some distance from the injection region. Therefore the electrons injected at the substorm onset drift past the riometer field-line to reach the satellites. The omega bands after 01:30 UT occur in the recovery phase of the substorm with expansion onset at $\sim 00: 30$ UT. This clearly coincides with the growth phase of the next substorm in the series that occurs at $\sim 02: 55$ UT (from the GOES data). Dispersionless signature are clear in the LANL data after 03:00 UT and it is likely that these are linked to the large increase in CNA observed by IRIS (at 03:00 UT in beam 36) that propagates across the field of view. The cessation of the Ps6 pulsation indicates that the omega-band current system breaks down after $\sim 03: 30$ UT at about the same time that the CNA starts to increase in the same location at the pulsation.

Unfortunately the LANL satellites are eastward of the location of IRIS and no trace of the Ps6 pulsation can be found in the data. However it is impossible to ascertain whether this is because no signature should be visible or whether it is simply because the omega band structure collapses before reaching the satellites. Inspection of the lower energy electron from the MPA (Magnetospheric Plasma Analyser) instrument (McComas et al., 1993) also reveals no pulsation. 


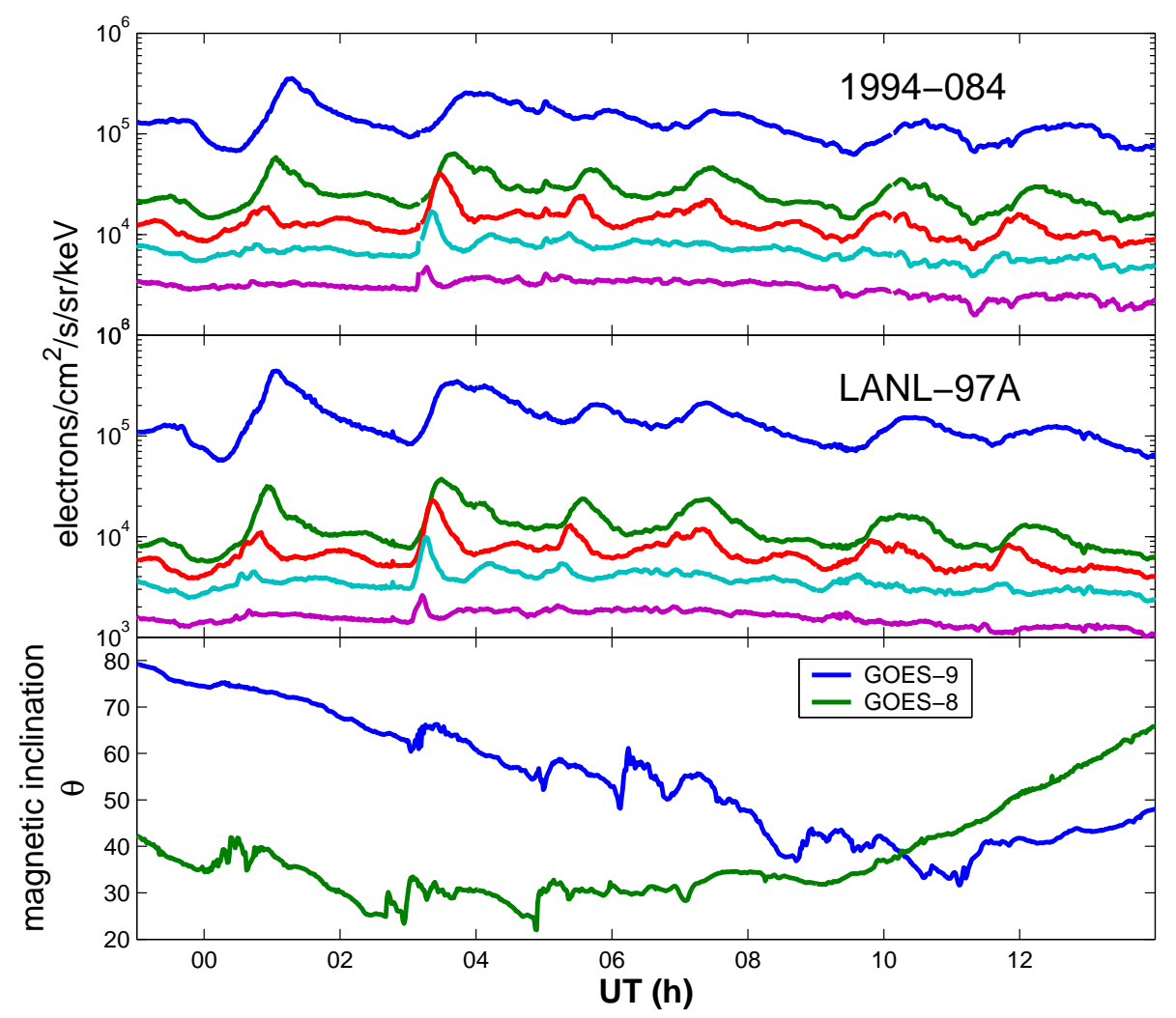

Fig. 6. Data from the LANL and GOES satellites on the 11 May 1998. The electron flux (LANL) is provided in seven channels: 50-75 keV (blue), $75-105 \mathrm{keV}$ (green), 105-150 keV (red), 150-225 keV (cyan) and 225-315 keV (magenta). The magnetic inclination is the angle of the local magnetic field to the equatorial plane; the larger the value of $\theta$ the more dipole-like the field is. The series of flux enhancements with dispersed energy signatures indicate periodic particle injections during substorms.

It is worth noting that ULF waves in the magnetosphere can modulate the observed geostationary electron flux either in a non-dispersed (e.g. field-line resonance) or a dispersed nature (e.g. drift bounce resonance). If the omega band structure does persist to the longitudes of the LANL satellites it is not having any such affect on the electron population. This suggests that the mechanism that modulates the energetic precipitation associated with omega bands is not associated with large scale ULF waves in the same way as for either field-line or drift-bounce resonance.

\subsection{Hall conductance and currents}

Wild et al. (2000) used VHF and HF radars in combination with magnetometer data to determine the equivalent current signatures and the ionospheric Hall conductance associated with the omega band. Senior et al. (2007) devised a statistical relationship between CNA and ionospheric conductance based upon IRIS data and estimates of the conductance derived from the EISCAT incoherent-scatter radar (e.g. Davies and Lester, 1999). Since the imaging riometer field of view essentially covers two of the magnetometers used in the previous study (SOR and TRO) we can make estimates of the
Hall conductance and compare with those derived by Wild et al. (2000).

For the local time period in question (04:00-06:00 MLT) the CNA-Hall conductance relationship is given by:

$\Sigma_{H}=37(\mathrm{CNA}-0.14)^{0.45}+4.3$

Where $\Sigma_{H}$ is the Hall conductance in Siemens. For values of CNA $<0.14 \mathrm{~dB}$ the $\Sigma_{H}$ is set to $4.3 \mathrm{~S}$. Applying this equation to the IRIS data provides estimates of the conductance that can be compared with those presented by Wild et al. (2000), which were based on magnetometer and radar measurements. It should be noted that there can be large uncertainties associated with any statistical relationship; this can be a particular problem when such a relationship, as outlined in Eq. (1), is applied to a case study. No statistical relationship can fully capture the details of a single event; however the relationship is useful for probing the omega band when considered alongside a different technique for determining conductance. We consider the limitations of the technique within the following discussion when compared with the results of Wild et al. (2000).

Figure 7 provides a direct comparison between the conductance estimated from IRIS beam 9 (a) and the estimates 


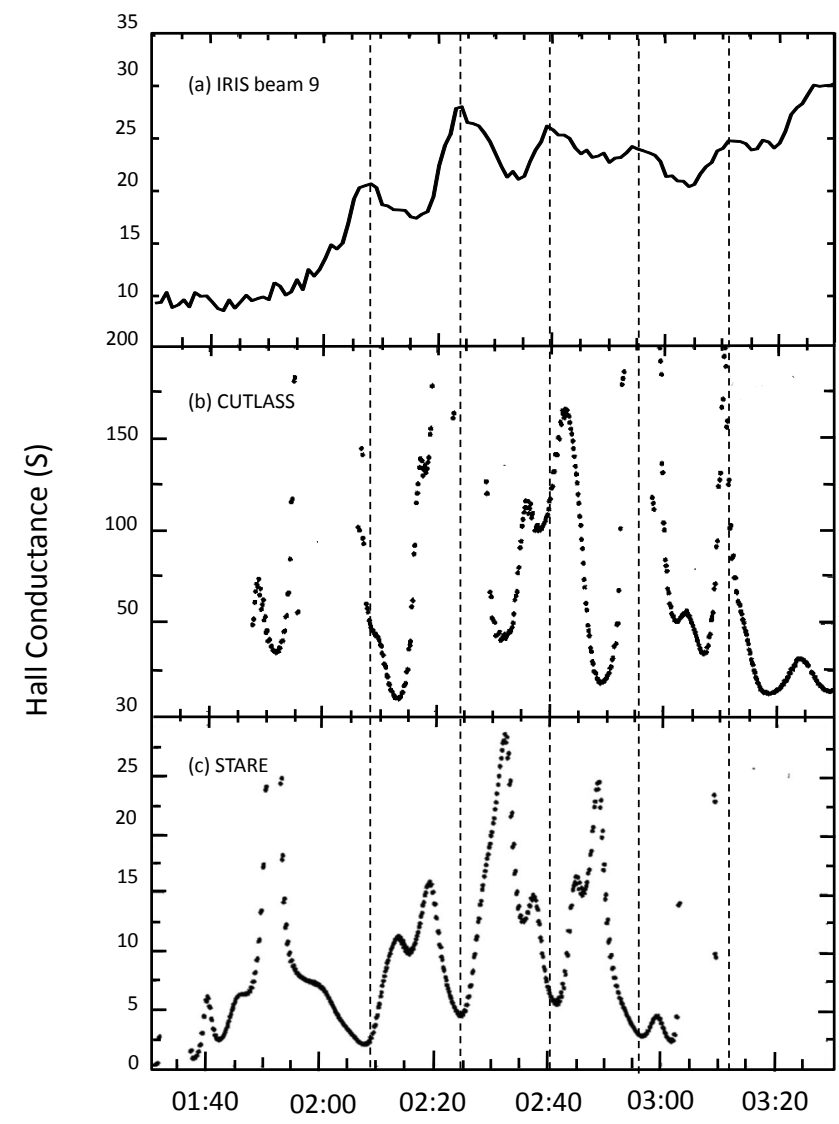

Fig. 7. A comparison of Hall Conductance estimates. Panel (a) is derived from Eq. (1) for data from beam 9 (over TRO) of IRIS. Panels (b) and (c) are taken from Wild et al. (2000) and correspond to estimates using the TRO magnetometer with beam 9 of CUTLASS and beam 4 of STARE respectively. Vertical dashed lines indicate the times of peak conductance according to IRIS. Peak magnitudes are comparable between (a) and (c) whereas the timing of peaks and troughs of the Ps6 match well between (a) and (b).

made by Wild et al. (2000) for TRO using CUTLASS (b) and STARE (c). The peak magnitudes from IRIS (20-30 S) are closer to those derived using STARE (c) than CUTLASS (b), though without the deep minima between peaks. The timing of the peaks and troughs in the conductance values better match with the estimates from the CUTLASS radar (b). The latter is likely a product of the experimental arrangement of the instruments; the relevant STARE beam was significantly off meridian and so was not orthogonal to the magnetic perturbation component. This may also help explain the discrepancy between the estimates of conductance from the two radars since the azimuthal velocity was significantly higher that the meridional. However this does not reconcile the difference in magnitude between the riometer estimate and the CUTLASS radar estimate (150-200 S at the peaks). As stated the riometer estimate is based upon the statistical relationship derived from comparisons between EISCAT data and IRIS (Senior et al., 2007); in that study conductance values did not reach the values stated in Wild et al. (2000) at any time (though the data did cover a number of substorm events). The difference could be due to a general inability of the CNA-Hall conductance relationship to reproduce conductance within an omega band. One possibility is a large change in the spectrum of precipitation. Lyons and Fennell (1986) showed that the spectrum of precipitation during an omega band includes a peak in low energy $(<1 \mathrm{keV})$ electrons but that the precipitation is still dominated by the more energetic electrons. This lower energy regime is more closely linked to changes in the Pederson conductance as outlined in Senior et al. (2007), any effect on the Hall conductance due to this peak is likely to be small. This does not exclude the possibility of a specific change in the electron spectrum at mid-energies (e.g. 10-20 keV) that would enhance the Hall conductance with only small affect (if any) on the CNA, resulting in an under-estimation of the conductance. Senior et al. (2008) compared different techniques for determining Hall and Pederson conductance using optical and riometer data with measurements from the EISCAT incoherent scatter radar during two intervals, one of which contained an omega band. The radar indicated Hall conductance with peak values of $60-80 \mathrm{~S}$ whereas the riometer estimates were $\sim 20 \mathrm{~S}$ less (25-30\% underestimate). It was noted that the statistical relationship underestimated the conductance in other periods when electron spectra derived from EISCAT measurements showed peaks around $10 \mathrm{keV}$. In the current event the CUTLASS-magnetometer values were $\sim 6-7$ times greater than the IRIS estimates in the pulsation peaks (whilst only slightly increased over IRIS in the troughs). It is far from clear that this large difference ( $80-85 \%$ difference) can be explained away by increased electron flux at the lower energies.

In Fig. 8 (top panel a) estimates of conductance are provided from riometer beams that correspond to TRO and the other magnetometer sites, SOR, TRO, KIL and ABK. In each case there is an increase in Hall conductance associated with the increased electron precipitation. For the beams in the northern part of the array (SOR, TRO and KIL) the omega band structure is clear. Values for SOR follow the same pattern as for TRO; comparison with the estimates made by Wild et al. (2000) show similar magnitudes as for the STARE-magnetometer pairing and similar timing for the CUTLASS-magnetometer pairing.

Panel (b) of Fig. 8 shows the ratio of the vertical to the horizontal magnetic perturbation for each magnetometer. This provides an indication of the location of the equivalent horizontal current within the omega band system. A sheet current in the ionosphere induces a horizontal magnetic field perturbation orthogonal to the direction of current flow detected by a magnetometer located on the ground under the sheet. Vertical magnetic perturbations cancel as long as the current density is uniform within the field of view of the magnetometer; if the sheet current is non-uniform, either by a reduction 

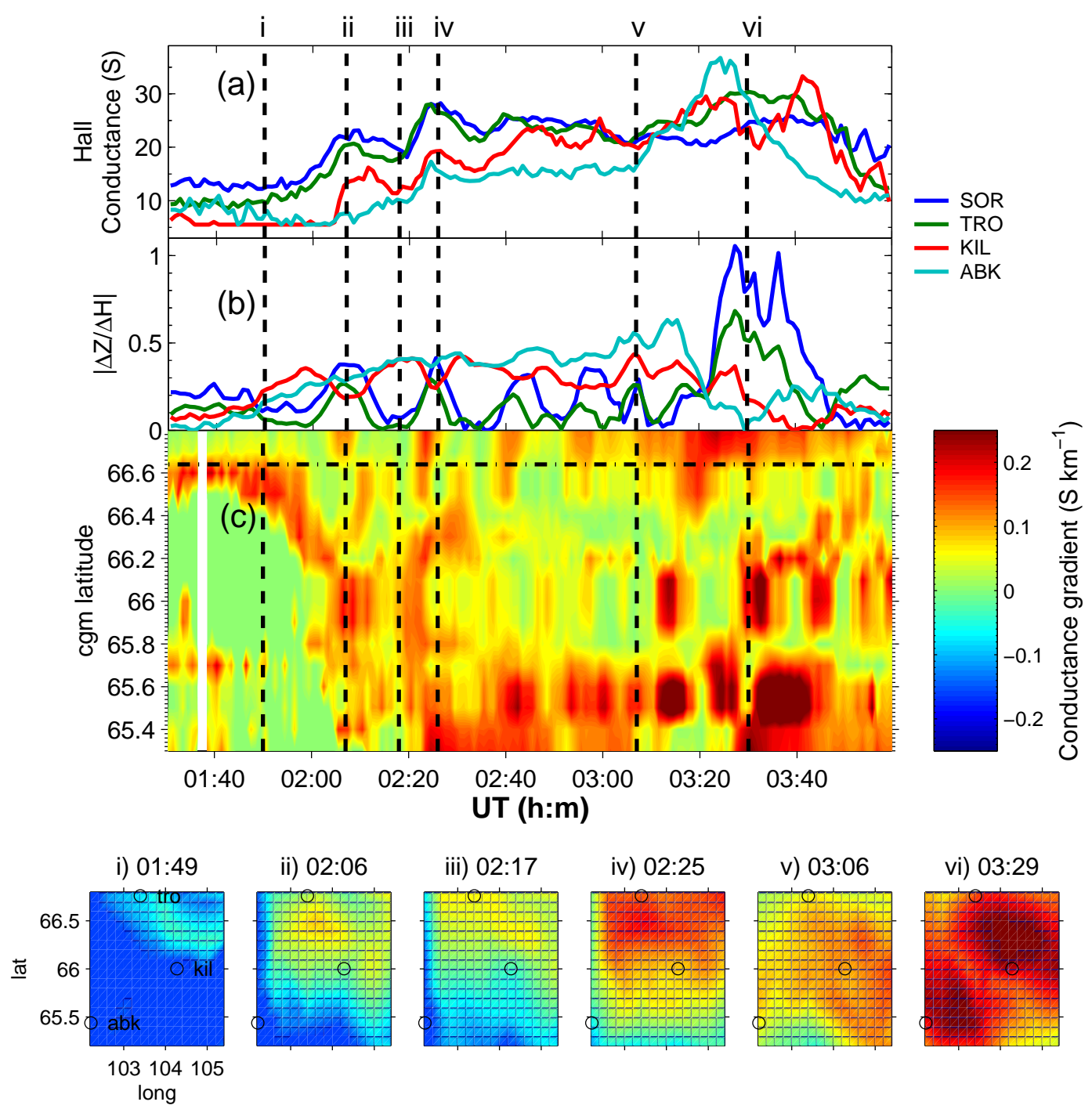

iii) $02: 17$

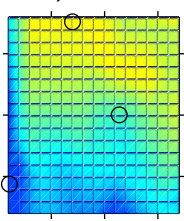

iv) $02: 25$

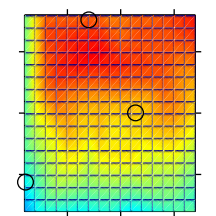

v) 03:06

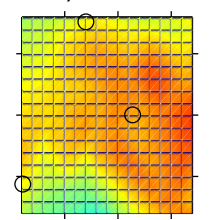

vi) $03: 29$

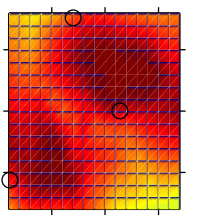

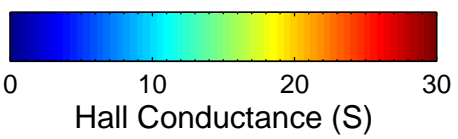

Fig. 8. (a) Estimates of Hall conductance from IRIS for beams closest to the indicated magnetometers. (b) The magnitude of dX/dZ (ratio of the vertical and horizontal perturbations) derived for the four magnetometers. (c) Keogram (at the cgm longitude of TRO) showing the longitudinal gradient in the conductance. The horizontal dash-dot line indicates the latitude of TRO. The bottom panels show instantaneous conductance maps derived from IRIS measurements for 6 selected intervals including: i) efore the Ps6; ii)-iv) peak-trough-peak through a cycle, v) the end of the pulsation; vi) during the subsequent precipitation event.

in current density (to zero, for example) or shear in the current flow, vertical magnetic perturbations will be detected on the ground. This is obviously the case where the Hall current circulates around a region of FAC and explains the observation that the $\mathrm{Z}$ component tends to peak at the location of FAC (e.g. Amm et al., 2005). Taking the ratio of the vertical and horizontal perturbations $(d Z / d X)$ provides an indication of the extent of the equivalent current system: if the magnetometer is located under a region of uniform current then $d Z / d X \sim 0$, whereas if the magnetometer approaches the edge of a current system, or is under a region of reducing current density, then $|d Z / d X|>0$. Thus $|d Z / d X|$ can be used as an indicator of the location of a particular magnetometer relative to the overhead current system. When used in conjunction with the estimates of conductance, provided by IRIS, it allows us to better characterise the extent of the omega band system by indicating where the currents are flowing within and around the precipitation features.

Figure 8 shows that the peaks in conductance (as derived from the riometer) for SOR and TRO coincide with 
the largest deviations in $|d Z / d X|$ suggesting that the horizontal sheet current is less uniform in the brightest part of the aurora. There is a small off-set between the peaks in conductance at KIL and when $|d Z / d X|$ tends back to zero; this is consistent with Hall current flowing at the flanks of the region of most intense precipitation which contains the upward FAC.

Panel (c) is a keogram of the latitudinal gradient in the conductance (in $\mathrm{S} \mathrm{km}^{-1}$ ), derived from IRIS and measured at the longitude of the TRO magnetometer $\left(102.9^{\circ}\right)$. It is immediately clear that at the latitude of TRO (the dash-dot line) that the peaks in conductivity occur with the largest deviations from zero in $|d Z / d X|$ and in regions of smallest conductance gradient. This can also be seen in the field-ofview plots (i-vi) in Fig. 8, which provide snapshots of the estimated Hall conductance in the IRIS field of view at six different intervals ( 1 min averages), marked by black dashed lines in panels $(\mathrm{a}-\mathrm{c})$. Three of the magnetometer positions (TRO, KIL and ABK) are marked on, SOR is located just beyond the northern edge of the field of view when converted to geomagnetic coordinates. A cycle of a Ps6 pulsation is shown (ii-iv), although the estimated conductance does not drop much between ii and iii this is due to the increase in precipitation over which the pulsation occurs. The variation in conductance gradient between ii and iv show clearly the omega band structure, with smallest gradients in the peaks and troughs of the pulsation in the IRIS data. The largest conductance gradient is located on the flanks of the conductance where $|d Z / d X|$ tends to zero, suggesting that the Hall current is tending towards uniformity (hence away from the region of FAC and the edge of the current system.

As demonstrated by Amm et al. (2005) the omega band is not strictly stationary in time; however the gradients in the temporal signature identified from the magnetometer-radar pairings are nearly analogous to the azimuthal gradients identified by IRIS (due to the motion of the current system). The images show that the precipitation in the omega band moves successively equatorward; this can also be seen in panel (f) of Fig. 2. This is consistent with an expanding polar cap during a substorm growth phase. The steady deviation from zero of the $\mid d X / d Z /$ measured at $\mathrm{ABK}$ (and to a lesser extent at KIL) would then be attributable to the equatorward motion of the current system.

Figure 6 shows that there was an injection of electrons and co-incident dipolarization of the geostationary magnetic field at 03:00 UT, which occurred as part of a series of injection events (e.g. a sawtooth event). This is indicative of a substorm expansion phase onset and is in agreement with the ground measurements presented by Wild et al. (2000). As stated earlier the recovery phase of the preceding substorm (which contains the omega band) overlapped the growth phase of this substorm where open flux is being added to the polar cap. The system breaks down at the onset of the new expansion phase when the field is re-ordered and the omega bands are replaced by gradient-drifting electrons that are freshly injected into the inner magnetosphere and precipitate as they drift.

\section{Conclusions}

The precipitation boundary of a substorm-related omegaband has been identified in imaging riometer data for the first time; this demonstrates the utility of the imaging riometer in the absence of optical measurements. Past observations of eastward-propagating absorption patches in the morning sector display similar characteristics and so Omega-band current structures might explain some of the quasi-periodicity observed in dawn side CNA.

Investigations into the ionospheric electrodynamics associated with auroral forms such as omega bands can be enhanced with the use of imaging riometers. In the absence of optical measurements the riometer provides a method of identifying the region of electron precipitation with accuracy beyond the ability of a magnetometer chain, which is measuring the equivalent Hall currents linked to the FAC rather than the precipitation itself. Due to the nature of the measurement the narrow beams of the riometer allow for more accurate detection of the conductance boundaries associated with omega bands than can be obtained with magnetometers, though the statistical nature of the CNA-Hall conductance relationship means that the absolute values may be underestimated. The combination of riometer and optical measurements would improve the conductance estimates (e.g. Senior et al., 2008).

Through the use of a statistical relationship between cosmic noise absorption and hall conductance the imaging riometer provides maps of conductance and estimates of the spatial gradients in the field of view. Although only a statistical estimate the derived values agree reasonably well with the calculations based upon magnetometer and VHF radar data for the same event, but disagree strongly with estimates using HF radars. It is not clear why such a large discrepancy occurs for the interval presented in this paper, but it may be due to strong enhancements of the electron flux at energies around $10 \mathrm{keV}$ to which the riometer is less sensitive, whilst the smaller values associated with the VHF radar may be due to the experimental arrangement of the instruments.

The omega band current system of 11 May 1998 occurred during one of the "teeth" of a sawtooth event, providing further evidence that these comprise a series of substorms. The current system in question occurred in the recovery phase of one substorm, which overlapped the growth phase of the next, disappearing with the expansion phase onset to be replaced by a gradient-curvature drifting electron population with more diffuse precipitation.

Acknowledgements. The authors wish to thank A. Senior for valuable discussions and suggestions. A. J. Kavanagh is supported by a grant from the UK Science and Technology Facilities Council. IRIS is operated by the Department of Communications Systems 
at Lancaster University (UK) in collaboration with the Sodankylä Geophysical Observatory, and was funded by the Science and Technology Facilities Council (STFC). Magnetometer data was taken from the Sub-Auroral Magnetometer Network (SAMNET) and the International Monitor for Auroral Geomagnetic Effects (IMAGE). SAMNET is operated by the Department of Communications Systems at Lancaster University (UK) and was funded by the Science and Technology Facilities Council (STFC). We thank the institutes who maintain the IMAGE Magnetometer Array. We thank G. Reeves and the energetic particle team at Los Alamos National Laboratory for the SOPA data.

Topical Editor M. Pinnock thanks A. M. Jorgensen and two other anonymous referees for their help in evaluating this paper.

\section{References}

Akasofu, S.-I.: A study of auroral displays photographed from DMSP-2 satellite and from the Alaska meridian chain of stations, Space Sci. Rev., 16, 617-725, 1974.

Amm, O., Aksnes, A., Stadsnes, J., Østgaard, N., Vondrak, R. R., Germany, G. A., Lu, G., and Viljanen, A.: Mesoscale ionospheric electrodynamics of omega bands determined from ground-based electromagnetic and satellite optical observations, Ann. Geophys., 23, 325-342, 2005, http://www.ann-geophys.net/23/325/2005/.

Baker, D. N., Stauning, P., Hones, E. W., Higbie, P. R., and Belian, R. D.: Near equatorial high resolution measurements of electron precipitation at $\mathrm{L} \approx 6.6, \mathrm{~J}$. Geophys. Res., 86, 2295-2313, 1981.

Belian, R., Gisler, G., Cayton, T., and Christensen, R.: High-z energetic particles at geosynchronous orbit during the great solar proton event series of October 1989, J. Geophys. Res., 97, 1689716906, 1992.

Borovsky, J. E., Thomsen, M. F., Reeves, G. D., Liemohn, M. W., Kozyra, J. U., Clauer, R., and Singer H. J.: Global sawtooth oscillations of the magnetosphere during large storms, EOS Trans. Amer. Geophys. Soc., 82(47), F1077, 2001.

Browne, S., Hargreaves, J. K., and Honary, B.: An Imaging Riometer for Ionospheric Studies, Elect. Comm. Eng. J., 7, 209-217, 1995.

Davies, J. A. and Lester, M.: The relationship between electric fields, conductances and currents in the high-latitude ionosphere: a statistical study using EISCAT data, Ann. Geophys., 17, 43-52, 1999, http://www.ann-geophys.net/17/43/1999/.

Davison, S. J. and Orr, D.: A Global Pulsation Event with Conjugate Study, Planet. Space Sci., 37, 253-267, 1989.

Greenwald, R. A., Baker, K. B., Dudeney, J. R., Pinnock, M., Jones, T. B., Thomas, E. C., Villain, J. P., Cerisier, J. C., Senior, C., Hanuise, C., Hunsucker, R. D., Sofko, G., Koehler, J., Nelsen, E., Pallinen, R.,Walker, A. D. M., Sato, N., and Yamagishi, H.: DARN/SuperDARN: A global view of the dynamics of he highlatitude convection, Space Sci. Rev., 71, 761-796, 1995.

Greenwald, R. A., Weiss, W., and Nielsen, E.: STARE: a new radar auroral backscatter experiment in northern Scandinavia, Radio Sci., 13, 1021-1039, 1978.

Gustafsson, G., Baumjohann, W., and Iversen, I.: Multi-method observations and modeling of the three-dimensional currents associated with a very strong Ps6 event, J. Geophys., 49, 138-145, 1981
Hargreaves, J. K., Browne, S., Ranta, H., Ranta, A., Rosenberg, T. J., and Detrick, D. L.: A study of substorm associated nightside spike events in auroral absorption using imaging riometers at South Pole and Kilpisjärvi, J. Atmos., Solar Terr. Phys., 59, 853-872, 1997.

Hargreaves, J. K., Chivers, H. J. A., and Axford, W. J.: Development of substorm in auroral radio absorption, Planet Space Sci., 23, 905-911, 1975.

Hargreaves, J. K. and Berry, M. G.: The eastward movement of the structure of auroral radio absorption events in the morning sector, Ann. Geophys., 32, 401-406, 1976.

Henderson, M. G., Friedel, R. H., Skoug, R. M., Jahn, J. M., Mende, S. B., Immel, T. J., Ingraham, J., Cayton, T. E., and Thomsen, M. F.: Simultaneous multipoint Observations of stormtime substorms with the CLUSTER, IMAGE, POLAR, geosynchronous and GPS satellites, EOS Trans. Amer. Geophys. Soc., 83(19), S287, 2002.

Kavanagh, A. J., Honary, F., McCrea, I. W., Donovan, E., Woodfield, E. E., Manninen, J., and Anderson, P. C.: Substorm related changes in precipitation in the dayside auroral zone - a multi instrument case study, Ann. Geophys., 20, 1321-1334, 2002, http://www.ann-geophys.net/20/1321/2002/.

Kavanagh, A. J., Lu, G., Donovan, E. F., Reeves, G. D., Honary, F., Manninen, J., and Immel, T. J.: Energetic electron precipitation during sawtooth injections, Ann. Geophys., 25, 1199-1214, 2007, http://www.ann-geophys.net/25/1199/2007/.

Kawasaki, K. and Rostoker, G.: Perturbation magnetic fields and current systems associated with eastward drifting auroral structures, J. Geophys. Res., 84, 1464-1480, 1979.

Little, C. G. and Leinbach, H.: The riometer: a device for the continuous measurements of Ionospheric absorption, Proc. IRE, 37, 315-320, 1959.

Lühr, H., Aylward, A., Bucher, S. C., Pajunpää, A., Pajunpää, K., Holmboe, T., and Zalewski, S. M.: Westward moving dynamic substorm features observed with the IMAGE magnetometer network and other ground-based instruments, Ann. Geophys., 16, 425-440, 1998, http://www.ann-geophys.net/16/425/1998/.

Lühr, H. and Schlegel, K.: Combined measurements of EISCAT and the EISCAT magnetometer cross to study omega bands, J. Geophys. Res., 99, 8951-8959, 1994.

Lyons, L. R. and Fennell, J. F.: Characteristics of Auroral Electron Precipitation on the Morningside, J. Geophys. Res., 91, 1122511234, 1986.

Makarevitch, R. A., Honary, F., McCrea, I. W., and Howells, V. S. C.: Imaging riometer observations of drifting absorption patches in the morning sector, Ann. Geophys., 22, 3461-3478, 2004, http://www.ann-geophys.net/22/3461/2004/.

McComas, D. J., Bame, S. J., Barraclough, B. L., Donart, J. R., Elphic, R. C., Gosling, J. T., Moldwin, M. B., Moore, K. R., and Thomsen, M. F.: Magnetospheric Plasma Analyzer (MPA): Initial Three-Spacecraft Observations from Geosynchronous Orbit, J. Geophys. Res., 98, 13453-13465, 1993.

Nielsen, E. and Honary, F.: Observations of ionospheric flows and particle precipitation following a Sudden Commencement, Ann. Geophys., 18, 908-917, 2000, http://www.ann-geophys.net/18/908/2000/.

Opgenoorth, H. J., Oksman, J., Kaila, K. U., Nielsen, E., and Baumjohann, W.: Characteristics of eastward drifting omega 
bands in the morning sector of the auroral oval, J. Geophys. Res., 88, 9171-9185, 1983.

Opgenoorth, H. J., Persson, M. A. L., Pulkkinen, T. I., and Pellinen, R. J.: Recovery phase of magnetospheric substorms and its association with morning sector aurora, J. Geophys. Res., 99, 4115-4129, 1994.

Ranta, H., Ranta, A., Collis, P. N., and Hargreaves, J. K.: Development of the Auroral Absorption Substorm: Studies of Pre-onset Phase and Sharp Onset Using an Extensive Riometer Network, Planet. Space Sci., 1287-1313, 1981.

Reeves, G., Belian, R., Cayton, T., Henderson, M., Christensen, R., McLachlan, P., and Ingraham, J.: Using los alamos geosynchronus energetic particle data in support of other missions, in: Satellite-Ground Based Coordination Source Book, edited by: Lockwood, M. and Opgenoorth, H., pp. 263-272, Eur. Space Agency, Noordwijk, The Netherlands, 1997

Rostoker, G. and Barichello, J. C.: Seasonal and diurnal variation of Ps 6 magnetic disturbances, J. Geophys. Res., 85, 161-163, 1980.

Saito, T.: Long-period irregular magnetic pulsations, Pi3, Space Sci. Rev., 21, 427-467, 1978.

Senior, A. and Honary, F.: Observations of the spatial structure of electron precipitation pulsations using an imaging riometer, Ann. Geophys., 21, 997-1003, 2003, http://www.ann-geophys.net/21/997/2003/.

Senior, A., Kavanagh, A. J., Kosch, M. J., and Honary, F.: Statistcial relationships between cosmic radio noise absorption and ionospheric electrical conductances in the auroral zone. J. Geophys. Res., 112, A11301, doi:10.1029/2007JA012519, 2007.
Senior, A., Kosch, M. J., and Honary, F.: Comparison of methods to determine auroral ionospheric conductances using ground-based optical and riometer data, Ann. Geophys., 26, 3831-3840, 2008, http://www.ann-geophys.net/26/3831/2008/.

Singer, H. J., Matheson, L., Grubb, R., Newman, A., and Bouwer, S. D.: Monitoring Space Weather with the GOES Magnetometers, SPIE Conference Proceedings, vol. 2812, p. 299-308, GOES-8 and Beyond, edited by: Washwell, E. R., 1996.

Solovyev, S. I., Baishev, D. G., Barkova, E. S., Engebretson, M. J., Posch, J. L., Hughes, W. J., Yumoto, K., and Pilipenko, V. A.: Structure of disturbances in the dayside and nightside ionosphere during periods of negative interplanetary magnetic field Bz, J. Geophys. Res., 104, 28019-28039, 1999.

Spanswick, E., Donovan, E., and Baker, G.: Pc5 modulation of high energy electron precipitation: particle interaction regions and scattering efficiency, Ann. Geophys., 23, 1533-1542, 2005, http://www.ann-geophys.net/23/1533/2005/.

Spanswick, E., Donovan, E., Friedel, R., and Korth, A.: Ground based identification of dispersionless electron injections, Geophys. Res. Lett., 34, L03101, doi:10.1029/2006GL028329, 2007.

Untiedt, J. and Baumjohann, W.: Studies of polar current systems using the IMS Scandinavian magnetometer array, Space Sci. Rev., 63, 245-390, 1993.

Wild, J. A., Yeoman, T. K., Eglitis, P., and Opgenoorth, H. J.: Multiinstrument observations of the electric and magnetic field structure of omega bands, Ann. Geophys., 18, 99-110, 2000, http://www.ann-geophys.net/18/99/2000/. 